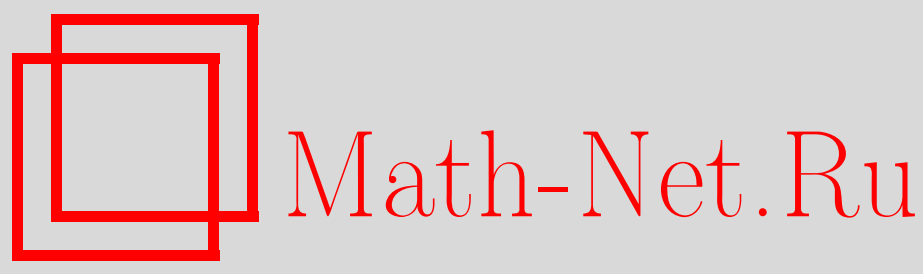

А. Я. Казаков, С. Ю. Славянов, Интегральные симметрии Эйлера для конфлюэнтного уравнения Гойна и симметрии уравнения Пенлеве PV, TMФ, 2014, том 179, номер 2, 189-195

DOI: https://doi.org/10.4213/tmf8634

Использование Общероссийского математического портала Math-Net.Ru подразумевает, что вы прочитали и согласны с пользовательским соглашением http: //www.mathnet.ru/rus/agreement

Параметры загрузки:

IP: 54.224 .135 .184

26 апреля 2023 г., 12:03:47

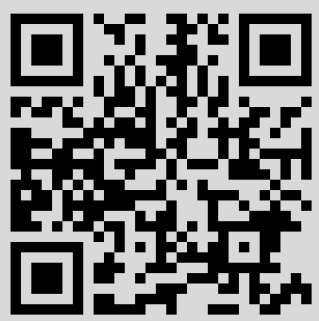


Том 179, № 2

май, 2014

(C) 2014 г. $\quad$ А. Я. Казаков ${ }^{* \dagger}$, С. Ю. Славянов ${ }^{\ddagger}$

\section{ИНТЕГРАЛЬНЫЕ СИММЕТРИИ ЭЙЛЕРА ДЛЯ КОНФЛЮЭНТНОГО УРАВНЕНИЯ ГОЙНА И СИММЕТРИИ УРАВНЕНИЯ ПЕНЛЕВЕ РV}

Интегральные симметрии Эйлера связывают между собой решения обыкновенных дифференциальных уравнений и порождают в некоторых случаях интегральные представления или соотношения между решениями связанных уравнений. Эти соотношения приводят к соответствующим симметриям матриц монодромии дифференциальных уравнений. Обсуждаются симметрии Эйлера для случая деформированного конфлюэнтного уравнения Гойна, которое, в свою очередь, связано с уравнением Пенлеве PV. Наличие симметрий для линейных уравнений ведет к соответствующей симметрии уравнения Пенлеве типа Окамото. Исходной точкой построений является выбор системы линейных уравнений, которая при редукции сводится к деформированному конфлюэнтному уравнению Гойна. Основная техническая проблема состоит в выборе биективного соответствия между параметрами системы и параметрами деформированного конфлюэнтного уравнения Гойна. Решение этой проблемы довольно громоздко, для него использовалась алгебраическая вычислительная система Maple.

Ключевые слова: конфлюэнтное уравнение Гойна, интегральное преобразование Эйлера, монодромия, ложная особая точка.

DOI: $10.4213 / \operatorname{tmf} 8634$

\section{1. ПРЕДВАРИТЕЛЬНЫЕ ЗАМЕЧАНИЯ}

Уравнение Гойна - это уравнение второго порядка с четырьмя регулярными особыми точками, в качестве которых мы выбираем $z_{1}=0, z_{2}=1, z_{3}=t, z_{4}=\infty$. Каноническая форма уравнения Гойна может быть выбрана следующим образом:

$$
w^{\prime \prime}+\left[\frac{1-\theta_{1}}{z}+\frac{1-\theta_{2}}{z-1}+\frac{1-\theta_{3}}{z-t}\right] w^{\prime}+\left[\frac{\alpha \beta}{z(z-1)}-\frac{t(t-1) H}{z(z-1)(z-t)}\right] w=0,
$$

* Санкт-Петербургский государственный университет технологии и дизайна, СанктПетербург, Россия. E-mail: a_kazak@mail.ru

${ }^{\dagger}$ Санкт-Петербургский государственный университет аэрокосмического приборостроения, Санкт-Петербург, Россия

${ }^{\ddagger}$ Санкт-Петербургский государственный университет, Санкт-Петербург, Россия. E-mail: slav@ss2034.spb.edu 
где $\theta_{j}, j=1,2,3,-$ характеристические экспоненты решений с сингулярностями в точках $z_{j}, z_{1}=0, z_{2}=1, z_{3}=t$, параметры $\alpha, \beta$ - характеристические экспоненты на бесконечности. Само это уравнение и различные его конфлюэнтные и специальные варианты широко используются в различных областях математической физики [1].

Деформированное уравнение Гойна, обозначаемое в работе [2] как Heun1, возникает из уравнения Гойна путем добавления ложной особенности в некоторой точке $z_{5}=\lambda, \lambda \neq 0,1, \tau$. Канонической формой уравнения Heun1 можно выбрать уравнение

$$
\begin{aligned}
w^{\prime \prime} & +w^{\prime}\left[\frac{1-\theta_{1}}{z}+\frac{1-\theta_{2}}{z-1}+\frac{1-\theta_{3}}{z-\tau}-\frac{1}{z-\lambda}\right]+ \\
& +w\left[\frac{\alpha \beta}{z(z-1)}+\frac{\lambda(\lambda-1) \mu}{z(z-1)(z-\lambda)}-\frac{\tau(\tau-1) H}{z(z-1)(z-\tau)}\right]=0 .
\end{aligned}
$$

В качестве набора параметров этого уравнения мы выбираем $\theta_{1}, \theta_{2}, \alpha, \beta, \tau, \lambda$ и $\mu$, причем $\theta_{3}$ рассматривается как зависимый параметр, поскольку должно выполняться условие Фукса, связывающее характеристические показатели в особых точках (выбор одного зависимого параметра $\theta_{3}$ среди $\theta_{1}, \theta_{2}$ и $\theta_{3}$ произволен). Параметр $H$ тоже не является независимым параметром уравнения (2): он определяется из условия, что точка $z=\lambda$ является ложной особой точкой. Это означает, что общее решение уравнения является голоморфной функцией в окрестности этой точки. Учет этого условия приводит к явному выражению для $H$ через параметры $\theta_{1}$, $\theta_{2}, \alpha, \beta, \lambda, \mu[3]$. Как было показано Окамото [4] (см. также [3], теорема А.5.2.13), при изменении $\lambda(\tau)$, где $\lambda(\tau)$ является решением уравнения PVI, уравнение Heun1 испытывает изомонодромную деформацию. Интегральные симметрии Эйлера для уравнения Heun1 и их связи с симметриями Окамото для уравнения PVI были изучены в работах [2], [5]. В работе [2] для этой цели было предложено использовать промежуточную линейную систему первого порядка.

Конфлюэнтное уравнение Гойна получается при конфлюэнции двух регулярных особых точек в одну иррегулярную особенность, которую обычно располагают на бесконечности. Этого можно достичь путем предельного перехода $\tau \rightarrow \infty, \theta_{3}=a \tau$. В результате мы приходим к уравнению

$$
w^{\prime \prime}+\left[a+\frac{\beta}{z}+\frac{\gamma}{z-1}\right] w^{\prime}+\left[\frac{a \sigma}{(z-1)}+\frac{a L}{z(z-1)}\right] w=0 .
$$

Это уравнение, так же как и его специальные случаи, играет ключевую роль во многих модельных задачах математической физики.

Под деформированным уравнением Гойна мы понимаем уравнение с двумя регулярными точками (они расположены в точках 0,1 ), одной иррегулярной особой точкой на бесконечности и одной добавленной ложной особой точкой. Такое уравнение мы обозначаем в дальнейшем Heunc1. Оно будет представлено в разделе 3.

В настоящей работе рассматриваются интегральные симметрии Эйлера для уравнения Heunc1, когда ядро интегрального преобразования - функция Эйлера $(z-t)^{\kappa}$ с подходящим $\kappa$. Такая симметрия, как было показано в [6], ведет к соотношениям между группами монодромии соответствующих уравнений. Хотя общая стратегия исследований была такая же, как в работе [2], технические трудности, к нашему удивлению, существенно возросли. В основном они связаны с правильным выбором промежуточной системы уравнений. 


\section{2. ИНТЕГРАЛЬНЫЕ СИММЕТРИИ ДЛЯ ЛИНЕЙНОЙ $(2 \times 2)$-МАТРИЧНОЙ СИСТЕМЫ ПЕРВОГО ПОРЯДКА}

Сначала рассмотрим интегральные симметрии Эйлера для следующей линейной системы первого порядка:

$$
\left(z^{2} A+z B+C\right) \frac{d W}{d z}=(\kappa z A+E) W
$$

где $A, B, C, E-(2 \times 2)$-матрицы, которые не зависят от $z, W(z)$ - двумерная векторфункция. Решение этой системы представляется как

$$
W(z)=\int_{L}(z-t)^{\kappa} V(t) d t
$$

где $V(t)$ - двумерная вектор-функция, контур интегрирования $L$ выбирается ниже. Подставляя функцию (5) в уравнение (4), получаем

$$
\begin{aligned}
\int_{L} d t & (z-t)^{\kappa}\left[\kappa \frac{z^{2} A+z B+C}{z-t}-(\kappa z A+E)\right] V(t)= \\
& =\int_{L} d t(z-t)^{\kappa}\left[\kappa \frac{\left(z^{2}-t^{2}\right) A+(z-t) B+t^{2} A+t B+C}{z-t}-(\kappa z A+E)\right] V(t)= \\
& =\int_{L} d t(z-t)^{\kappa}\left[\kappa \frac{t^{2} A+t B+C}{z-t}+\kappa t A+\kappa B-E\right] V(t)=I .
\end{aligned}
$$

Заметим, что $\kappa(z-t)^{\kappa-1}=-\frac{d}{d t}(z-t)^{\kappa}$. Следовательно, интегрируя по частям в последнем интеграле (в предположении, что внеинтегральные члены пропадают), находим

$$
\begin{aligned}
I & =\int_{L}\left\{\frac{d}{d t}\left[\left(t^{2} A+t B+C\right) V(t)\right]+(\kappa t A+\kappa B-E) V(t)\right\}(z-t)^{\kappa} d t= \\
& =\int_{L}\left\{\left(t^{2} A+t B+C\right) \frac{d V(t)}{d t}+[(\kappa+2) t A+(\kappa+1) B-E] V(t)\right\}(z-t)^{\kappa} d t .
\end{aligned}
$$

Если $V(t)$ - решение системы

$$
\left(t^{2} A+t B+C\right) \frac{d V(t)}{d t}=[-(\kappa+2) t A+E-(\kappa+1) B] V(t)=0
$$

то выражение под интегралом исчезает. Выбор контура интегрирования $L$ и решения уравнения (7) $V(t)$, гарантирующие исчезновение внеинтегральных членов в уравнении (6), определяются следующим образом. Система уравнений (7) имеет в качестве регулярных особых точек нули функции $\operatorname{det}\left(t^{2} A+t B+C\right)$ (мы здесь полагаем, что нет случайных вырождений). В качестве $V(t)$ мы выбираем любое решение, ветвящееся в окрестности какой-либо регулярной особой точки $t_{*}$ системы (7), а в качестве $L$ - двойную восьмерку, охватывающую точки $t=t_{*}$ и $t=z$. Этот выбор приводит к исчезновению внеинтегральных членов при интегрировании по частям. 
Обращая наши выкладки, приходим к следующему выводу. Если $V(t)$ - описанное выше решение системы (7), а $L$ - описанный выше контур интегрирования, то вектор-функция $W(z)$, определенная интегралом (5), является решением системы (4).

Интегральная симметрия Эйлера (5) связывает, таким образом, решения систем (4) и $(7)$, причем если $V(t)$ ветвится в точке $t=t_{*}$, то соответствующая векторфункция $W(z)$ ветвится в точке $z=z_{*}, z_{*}=t_{*}$.

Аналитическое продолжение таких решений дает возможность получить соответствующие симметрии для матриц монодромии решений (подробнее см. [6], [7]).

\section{3. СООТНОШЕНИЯ МЕЖДУ ПАРАМЕТРАМИ УРАВНЕНИЙ ВТОРОГО ПОРЯДКА}

Хорошо известно, что линейная система двух уравнений первого порядка может быть сведена к одному линейному уравнению второго порядка. Согласно интегральному представлению (5) справедливы соответствующие уравнения и для соответствующих компонент вектор-функций $W(z), V(t)$. Преобразуя систему (4) к уравнению для функции $w(z)=w_{1}(z)$, мы получаем

$$
w(z)=\int_{L}(z-t)^{\kappa} v(t) d t
$$

$v(t)=v_{1}(t)$. В дальнейшем параметры системы (4) выбираются таким образом, чтобы уравнения для $w(z), v(z)$ совпадали с Heunc1.

Мы используем следующую каноническую параметризацию для уравнения Неunc1:

$$
u^{\prime \prime}(z)+\left[a+\frac{\beta}{z}+\frac{\gamma}{z-1}-\frac{1}{z-\lambda}\right] u^{\prime}(z)+\frac{1}{z(z-1)}\left[\sigma a z+a L+\frac{\chi \lambda(\lambda-1)}{z-\lambda}\right] u(z)=0 .
$$

Это уравнение содержит шесть произвольных параметров $a, \beta, \gamma, \lambda, \sigma, \chi$, параметр $L$ определяется из условия, что точка $z=\lambda$ является ложной особенностью. Ниже для большей компактности формул мы используем обозначения

$$
P_{0}=z(z-1), \quad P_{1}=a z(z-1)+\beta(z-1)+\gamma z \text {. }
$$

В этих обозначениях первоначальное уравнение принимает вид

$$
P_{0}(z) y^{\prime \prime}+\left(P_{1}(z)-\frac{1}{z-\lambda}\right) y^{\prime}+\frac{1}{z(z-1)}\left(\sigma a z+a L+\frac{\chi \lambda(\lambda-1)}{z-\lambda}\right) y=0,
$$

а выражение для $L-$ вид

$$
L=\chi\left[P_{0}^{\prime}(\lambda)-P_{1}(\lambda)-\chi P_{0}(\lambda)\right]-\sigma a \lambda
$$

Для того чтобы получить уравнение (9) при редукции системы (4), мы выбрали следующую параметризацию матричных коэффициентов в (4):

$$
A=\left(\begin{array}{ll}
1 & 0 \\
0 & 0
\end{array}\right), \quad B=\left(\begin{array}{ll}
0 & 1 \\
1 & 0
\end{array}\right), \quad C=\left(\begin{array}{cc}
2 c+1 & -2 c-1 \\
c & -c
\end{array}\right), \quad E=\left(\begin{array}{ll}
e_{1} & e_{2} \\
e_{3} & e_{4}
\end{array}\right) .
$$


Эта параметризация выглядит слегка икусственной и может быть видоизменена. Однако более общая параметризация приводит к неимоверному росту вычислений, делая их нечитаемыми, в то время как данная простая параметризация уменьшает число независимых параметров. Для дальнейших упрощений можно ввести три вектора

$$
\mathbf{C}=(2 c+1, c), \quad \mathbf{E}_{\mathbf{1}}=\left(e_{1}, e_{3}\right), \quad \mathbf{E}_{\mathbf{2}}=\left(e_{2}, e_{4}\right)
$$

с внешними произведениями $\mathbf{C} \wedge \mathbf{E}_{\mathbf{1}}, \mathbf{C} \wedge \mathbf{E}_{\mathbf{2}}$. В выбранном случае система (4) записывается как

$$
\left(\begin{array}{cc}
z^{2}+2 c+1 & z-2 c-1 \\
z+c & -c
\end{array}\right) W^{\prime}=\left(\begin{array}{cc}
\kappa z+e_{1} & e_{2} \\
e_{3} & e_{4}
\end{array}\right) W
$$

Она может быть преобразована к следующей форме:

$$
\begin{gathered}
D(z) W^{\prime}=T(z) W \\
D(z)=-(c+1) z(z-1), \\
T(z)=\left(\begin{array}{cc}
\left(-c \kappa-e_{3}\right) z+\mathbf{C} \wedge \mathbf{E}_{\mathbf{1}} & -e_{4} z+\mathbf{C} \wedge \mathbf{E}_{\mathbf{2}} \\
\left(e_{3}-\kappa\right) z^{2}-\left(c \kappa+e_{1}\right) z+\mathbf{C} \wedge \mathbf{E}_{\mathbf{1}} & e_{4} z^{2}-e_{2} z+\mathbf{C} \wedge \mathbf{E}_{\mathbf{2}}
\end{array}\right) .
\end{gathered}
$$

Регулярные особые точки этой системы суть нули функции $D(z)$. Исключив $w_{2}(z)$ из нашей системы, мы получим для функции $w(z)=w_{1}(z)$ уравнение второго порядка

$$
w^{\prime \prime}(z)+M(z) w^{\prime}(z)+N(z) w(z)=0
$$

где

$$
\begin{gathered}
M(z)=\frac{D^{\prime}(z)-T_{11}(z)-T_{22}(z)}{D(z)}-\frac{T_{12}^{\prime}(z)}{T_{12}(z)}, \\
N(z)=\frac{\operatorname{det} T}{D^{2}(z)}-\frac{T_{12}(z) T_{11}^{\prime}(z)-T_{11}(z) T_{12}^{\prime}(z)}{D(z) T_{12}(z)} .
\end{gathered}
$$

Ясно, что особенности системы (12) будут также особенностями уравнения (13). Заметим, что $T_{12}(z)$ - полином первой степени по $z$. При исключении функции $w_{2}(z)$ и выводе уравнения для $w_{1}(z)$ мы получим ложную особенность первого порядка, которая является простым корнем функции $T_{12}(z)$ (этот факт обсуждается, например, в [8], лемма 6.1.2). Легко проверяется, что функция $\operatorname{det} T(z)$ принимает нулевые значения в точках $z=0,1$. Следовательно, функция $N(z)$ не имеет кратных корней. Таким образом, функция $w_{1}(z)$ является решением уравнения Heunc1.

Из наших вычислений следует, что ложная особенность находится в точке

$$
\lambda=\frac{\mathbf{C} \wedge \mathbf{E}_{\mathbf{2}}}{e_{4}},
$$

и $\kappa=-\sigma$. Приравнивая коэффициенты разложений в полюсах $M(z), N(z)$ и коэффициенты уравнения (9) с учетом (14), (15), мы находим соотношения между параметрами системы (12) и параметрами уравнения (9). Мы опускаем соответствующие громоздкие вычисления, которые могут быть проведены с помощью программы Maple. 
Как уже отмечалось, мы получаем уравнение (9) для функции $w(z)=w_{1}(z)$ при принятом выборе параметров в (12). Далее система (7) принимает вид

$$
D(t) V^{\prime}(t)=\widetilde{P}(t) V(t)
$$

где

$$
\begin{gathered}
\widetilde{P}_{11}(t)=[\kappa(c+1)+2 c+1] t+\mathbf{C} \wedge \mathbf{E}_{\mathbf{1}}-(2 c+1)(\kappa+1), \\
\widetilde{P}_{12}(t)=-e_{4} t+\mathbf{C} \wedge \mathbf{E}_{\mathbf{2}}+c(\kappa+1), \\
\widetilde{P}_{21}(t)=\left(1+e_{3}\right) t^{2}+\left(c \kappa+2 c-e_{1}\right) t+\mathbf{C} \wedge \mathbf{E}_{\mathbf{1}}-(2 c+1)(\kappa+1), \\
\widetilde{P}_{22}(t)=e_{4} t^{2}+\left(\kappa+1-e_{2}\right) t+c(\kappa+1)+\mathbf{C} \wedge \mathbf{E}_{\mathbf{2}} .
\end{gathered}
$$

Исключив $v_{2}(t)$, мы придем к уравнению второго порядка для $v(t)=v_{1}(t)$ :

$$
v^{\prime \prime}(t)+\widetilde{M}(t) v^{\prime}(t)+\widetilde{N}(t) v(t)=0 .
$$

Заметим, что $\widetilde{P}_{12}(t)$ - полином первой степени по $t$. Следовательно, его нуль генерирует одну ложную особую точку. Взяв явные выражения для $\widetilde{M}(t), \widetilde{N}(t)$, мы получим для функции $v(t)$ уравнение Heunc1:

$$
v^{\prime \prime}(t)+\left[\widetilde{a}+\frac{\widetilde{\beta}}{t}+\frac{\widetilde{\gamma}}{t-1}-\frac{1}{t-\widetilde{\lambda}}\right] v^{\prime}(t)+\frac{1}{t(t-1)}\left[\tilde{\sigma} \widetilde{a} t+\widetilde{a} \widetilde{L}+\frac{\tilde{\chi} \widetilde{\lambda}(\widetilde{\lambda}-1)}{t-\widetilde{\lambda}}\right] v(t)=0
$$

где

$$
\begin{aligned}
\widetilde{a}=a, \quad \tilde{\beta} & =\beta+1-\sigma, \quad \widetilde{\gamma}=\gamma+1-\sigma, \quad \tilde{\sigma}=2-\sigma, \\
\tilde{\lambda} & =\lambda+\frac{(1-\sigma) P_{0}(\lambda)}{P_{1}(\lambda)-P_{0}^{\prime}(\lambda)+\chi P_{0}(\lambda)}, \\
\widetilde{L} & =\widetilde{\chi}\left[P_{0}^{\prime}(\tilde{\lambda})-P_{1}(\widetilde{\lambda})-\tilde{\chi} P_{0}(\widetilde{\lambda})\right]-a \tilde{\sigma} \widetilde{\lambda} .
\end{aligned}
$$

Введем для упрощения обозначение $\beta+\gamma=\delta$. Тогда

$$
\begin{aligned}
\tilde{\chi}= & \frac{1}{\left(\chi P_{0}(\lambda)+P_{1}(\lambda)-\lambda(\sigma+1)+1\right)\left(\chi P_{0}(\lambda)+P_{1}(\lambda)-\lambda(\sigma+1)+\sigma\right)} \times \\
& \times\left\{\chi P_{1}^{2}(\lambda)+\chi^{2}(\chi+2 a) P_{0}^{2}(\lambda)+2 \lambda\left[\chi^{2}(\delta-2) P_{0}(\lambda)+(a \sigma+\sigma \chi-a-3 \chi) P_{1}(\lambda)\right]+\right. \\
& +P_{1}(\lambda)\left(-2 \sigma^{2}-\sigma \chi-a \sigma+4-2 \sigma+a-\delta+3 \chi+\delta \sigma\right)- \\
& -P_{0}(\lambda) \chi\left(2 \beta \chi-2 \chi-\delta+\sigma^{2}+\delta \sigma-5\right)+ \\
& +\lambda\left(\sigma^{2} \delta+2 \sigma^{2}-\delta-2-\beta \chi+\beta \sigma \chi+\gamma \chi-\sigma \chi \gamma\right)+ \\
& \left.+1+2 \beta+\chi-\sigma^{2} \beta+a-\gamma-a \sigma+\sigma \gamma-\sigma \beta \chi-\sigma \beta+\beta \chi-\sigma^{2}\right\}
\end{aligned}
$$

Заметим, что соотношения (20) могут быть непосредственно получены из интегральной связи (8). Представленные соотношения задают интегральную симметрию уравнения Heunc1. Решения уравнения Heunc1 с параметрами $a, \beta, \gamma, \lambda, \sigma, \chi$ с помощью соотношения (8) выражаются в терминах решений уравнения Неunc1 с параметрами $\widetilde{a}, \widetilde{\beta}, \widetilde{\gamma}, \widetilde{\lambda}, \widetilde{\sigma}, \widetilde{\chi}$. 
Эти соотношения обладают следующими замечательными свойствами.

1. Они дают рациональные выражения для параметров нового уравнения $\widetilde{a}, \widetilde{\beta}, \widetilde{\gamma}$, $\widetilde{\lambda}, \widetilde{\sigma}, \tilde{\chi}$ через параметры исходного уравнения $a, \beta, \gamma, \lambda, \sigma, \chi$.

2. Явные вычисления (с помощью программы Maple) показывают, что повторное использование этих соотношений ведет к тождеству. Это означает, что эти два преобразования находятся в инволюции. Ясно, что этот факт также следует из прямого сравнения (4) и (7).

3. Как показано в работе [6], интегральные соотношения изученного типа определяют соотношения между матрицами монодромии для соответствующих дифференциальных уравнений. Следовательно, если коэффициенты исходного уравнения (9) испытывают изомонодромные деформации, то коэффициенты преобразованного дифференциального уравнения (19) также испытывают изомонодромные деформации. Изомонодромные деформации уравнения (9) эквивалентны уравнению Пенлеве PV, следовательно, соотношения (20)-(22) ведут к симметриям решений уравнения PV. Эти симметрии описываются рациональными функциями, и, следовательно, они являются симметриями типа Окамото.

Почему нужна промежуточная $(2 \times 2)$-матричная система? Почему не применить преобразование Эйлера к первоначальному уравнению второго порядка - конфлюэнтному уравнению Гойна без добавленной ложной особой точки? $\mathrm{K}$ сожалению, простые вычисления показывают, что результатом такого преобразования будет дифференциальное уравнение четвертого порядка, которое в общей ситуации имеет гораздо больше свободных параметров.

В заключение отметим, что мы описали симметрии преобразования Эйлера для уравнений Heun1 [2] и Heunc1. Вопрос об аналоге таких соотношений для биконфлюэнтных и дважды конфлюэнтных деформированных уравнений Гойна остается открытым.

\section{Список литературы}

[1] S. Yu. Slavyanov, W. Lay, Special Functions. A Unified Theory Based on Singularities, Oxford Univ. Press, Oxford, 2000.

[2] А. Я. Казаков, С. Ю. Славянов, ТМФ, 155:2 (2008), 252-264.

[3] Y. Sibuya, Linear Differential Equations in the Complex Domain: Problems of Analytic Continuation, Translations of Mathematical Monographs, 82, AMS, Providence, RI, 1990.

[4] K. Okamoto, J. Fac. Sci. Univ. Tokyo Sect. IA Math., 33:3 (1986), 575-618.

[5] K. Takemura, SIGMA, 5 (2009), 040, 22 pp.

[6] А. Я. Казаков, ТМФ, 116:3 (1998), 323-335.

[7] A. Ya. Kazakov, J. Phys. A, 39:10 (2006), 2339-2348.

[8] K. Iwasaki, H. Kimura, S. Shimomura, M. Yosida, From Gauss to Painlevé: A Modern Theory of Special Functions, Aspects of Mathematics, E16, Vieweg \& Sohn, Braunschweig, 1991. 